\title{
testthat: Get Started with Testing
}

\author{
by Hadley Wickham
}

\begin{abstract}
Software testing is important, but many of us don't do it because it is frustrating and boring. testthat is a new testing framework for $\mathrm{R}$ that is easy learn and use, and integrates with your existing workflow. This paper shows how, with illustrations from existing packages.
\end{abstract}

\section{Introduction}

Testing should be something that you do all the time, but it's normally painful and boring. testthat (Wickham, 2011) tries to make testing as painless as possible, so you do it as often as possible. To make that happen, testthat:

- Provides functions that make it easy to describe what you expect a function to do, including catching errors, warnings and messages.

- Easily integrates in your existing workflow, whether it's informal testing on the command line, building test suites, or using ' $R$ CMD check'.

- Can re-run tests automatically as you change your code or tests.

- Displays test progress visually, showing a pass, fail or error for every expectation. If you're using the terminal, it'll even colour the output.

testthat draws inspiration from the xUnit family of testing packages, as well from many of the innovative Ruby testing libraries like rspec ${ }^{1}$, testy ${ }^{2}$, bacon $^{3}$ and cucumber ${ }^{4}$. I have used what I think works for $\mathrm{R}$, and abandoned what doesn't, creating a testing environment that is philosophically centred in R.

\section{Why test?}

I wrote testthat because I discovered I was spending too much time recreating bugs that I had previously fixed. While I was writing the original code or fixing the bug, I'd perform many interactive tests to make sure the code worked, but I never had a system for retaining these tests and running them, again and again. I think this is a common development practice of R programmers: it's not that we don't test our code, it's that we don't store our tests so they can be re-run automatically.

\footnotetext{
$1_{\text {http: } / / \text { rspec.info/ }}$

2 http://github.com/ahoward/testy

${ }^{3}$ http://github.com/chneukirchen/bacon

${ }^{4}$ http://wiki.github.com/aslakhellesoy/cucumber/
}

In part, this is because existing $\mathrm{R}$ testing packages, such as RUnit (Burger et al., 2009) and svUnit (Grosjean, 2009), require a lot of up-front work to get started. One of the motivations of testthat is to make the initial effort as small as possible, so you can start off slowly and gradually ramp up the formality and rigour of your tests.

It will always require a little more work to turn your casual interactive tests into reproducible scripts: you can no longer visually inspect the output, so instead you have to write code that does the inspection for you. However, this is an investment in the future of your code that will pay off in:

- Decreased frustration. Whenever I'm working to a strict deadline I always seem to discover a bug in old code. Having to stop what I'm doing to fix the bug is a real pain. This happens less when I do more testing, and I can easily see which parts of my code I can be confident in by looking at how well they are tested.

- Better code structure. Code that's easy to test is usually better designed. I have found writing tests makes me extract out the complicated parts of my code into separate functions that work in isolation. These functions are easier to test, have less duplication, are easier to understand and are easier to re-combine in new ways.

- Less struggle to pick up development after a break. If you always finish a session of coding by creating a failing test (e.g. for the feature you want to implement next) it's easy to pick up where you left off: your tests let you know what to do next.

- Increased confidence when making changes. If you know that all major functionality has a test associated with it, you can confidently make big changes without worrying about accidentally breaking something. For me, this is particularly useful when I think of a simpler way to accomplish a task - often my simpler solution is only simpler because I've forgotten an important use case!

\section{Test structure}

testthat has a hierarchical structure made up of expectations, tests and contexts. 
- An expectation describes what the result of a computation should be. Does it have the right value and right class? Does it produce error messages when you expect it to? There are 11 types of built-in expectations.

- A test groups together multiple expectations to test one function, or tightly related functionality across multiple functions. A test is created with the test_that function.

- A context groups together multiple tests that test related functionality.

These are described in detail below. Expectations give you the tools to convert your visual, interactive experiments into reproducible scripts; tests and contexts are just ways of organising your expectations so that when something goes wrong you can easily track down the source of the problem.

\section{Expectations}

An expectation is the finest level of testing; it makes a binary assertion about whether or not a value is as you expect. An expectation is easy to read, since it is nearly a sentence already: expect_that $(a$, equals (b) ) reads as "I expect that a will equal b". If the expectation isn't true, testthat will raise an error.

There are 11 built-in expectations:

- equals() uses all.equal () to check for equality with numerical tolerance:

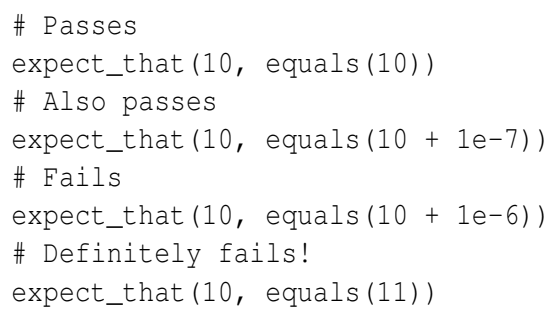

- is_identical_to() uses identical () to check for exact equality:

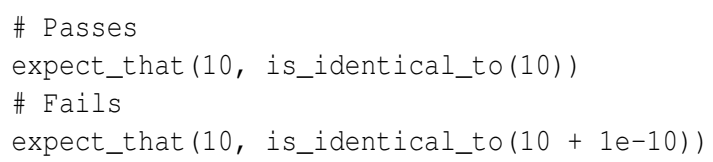

- is_equivalent_to() is a more relaxed version of equals () that ignores attributes:

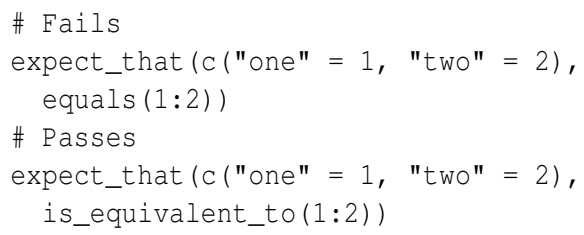

- is_a () checks that an object inherit () s from a specified class:

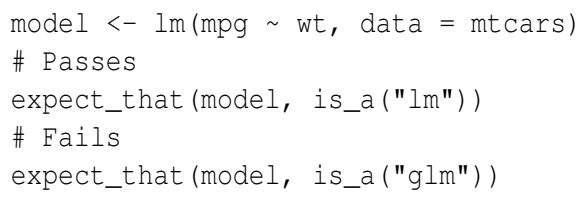

- matches () matches a character vector against a regular expression. The optional all argument controls where all elements or just one element need to match. This code is powered by str_detect () from the stringr (Wickham, 2010) package:

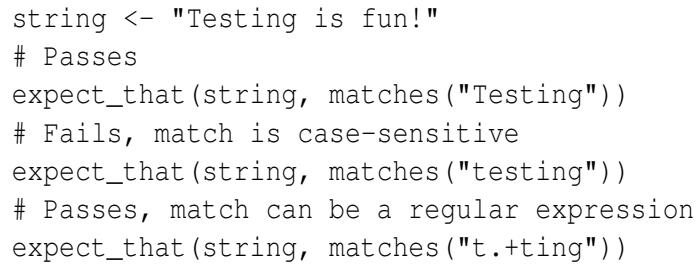

- prints_text() matches the printed output from an expression against a regular expression:

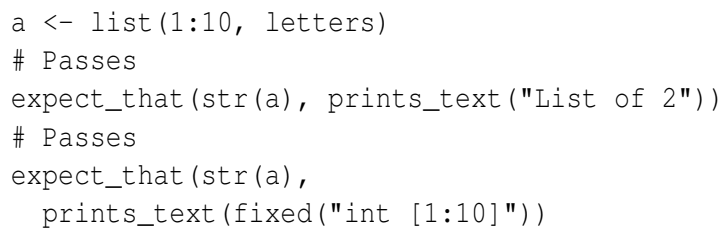

- shows_message() checks that an expression shows a message:

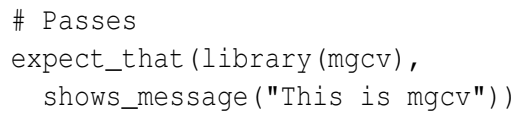

- gives_warning() expects that you get a warning:

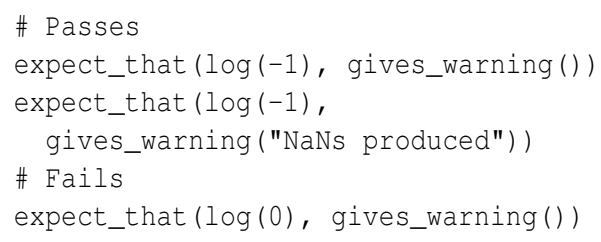

- throws_error() verifies that the expression throws an error. You can also supply a regular expression which is applied to the text of the error:

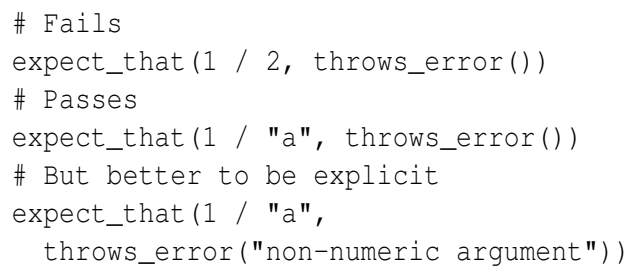




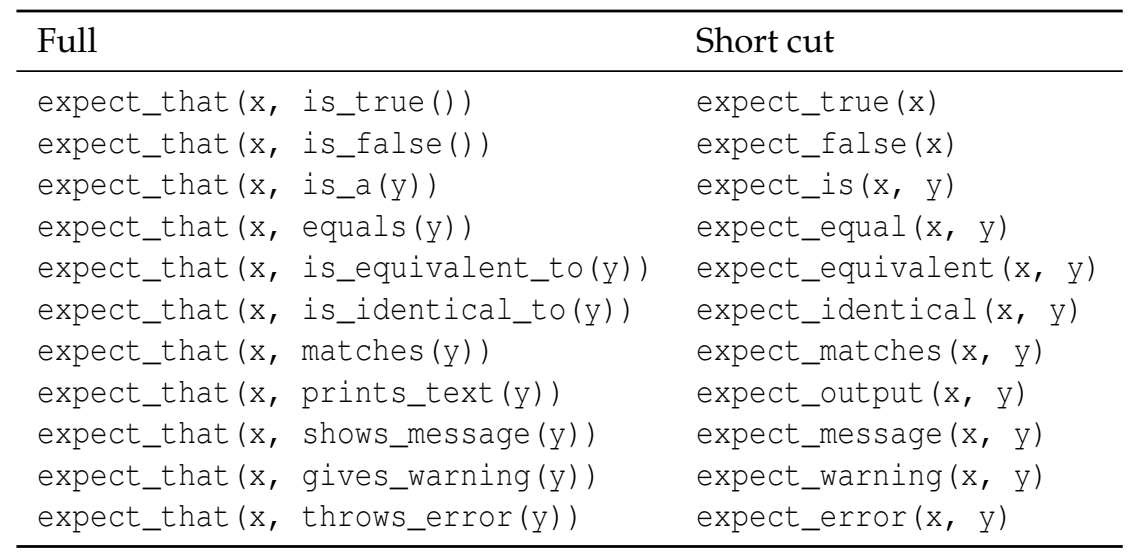

Table 1: Expectation shortcuts

- is_true() is a useful catchall if none of the other expectations do what you want - it checks that an expression is true. is_false() is the complement of is_true ().

If you don't like the readable, but verbose, expect_that style, you can use one of the shortcut functions described in Table 1.

You can also write your own expectations. An expectation should return a function that compares its input to the expected value and reports the result using expectation(). expectation () has two arguments: a boolean indicating the result of the test, and the message to display if the expectation fails. Your expectation function will be called by expect_that with a single argument: the actual value. The following code shows the simple is_true expectation. Most of the other expectations are equally simple, and if you want to write your own, I'd recommend reading the source code of testthat to see other examples.

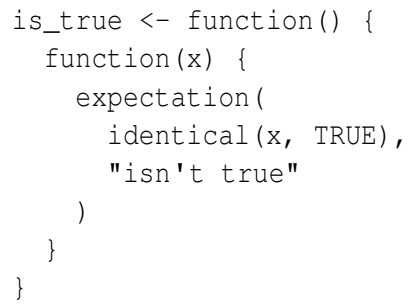

Running a sequence of expectations is useful because it ensures that your code behaves as expected. You could even use an expectation within a function to check that the inputs are what you expect. However, they're not so useful when something goes wrong: all you know is that something is not as expected, you know nothing about where the problem is. Tests, described next, organise expectations into coherent blocks that describe the overall goal of that set of expectations.

\section{Tests}

Each test should test a single item of functionality and have an informative name. The idea is that when a test fails, you should know exactly where to look for the problem in your code. You create a new test with test_that, with parameters name and code block. The test name should complete the sentence "Test that ..." and the code block should be a collection of expectations. When there's a failure, it's the test name that will help you figure out what's gone wrong.

Figure 1 shows one test of the floor_date function from lubridate (Wickham and Grolemund, 2010). There are 7 expectations that check the results of rounding a date down to the nearest second, minute, hour, etc. Note how we've defined a couple of helper functions to make the test more concise so you can easily see what changes in each expectation.

Each test is run in its own environment so it is self-contained. The exceptions are actions which have effects outside the local environment. These include things that affect:

- The filesystem: creating and deleting files, changing the working directory, etc.

- The search path: package loading \& detaching, attach.

- Global options, like options () and par().

When you use these actions in tests, you'll need to clean up after yourself. Many other testing packages have set-up and teardown methods that are run automatically before and after each test. These are not so important with testthat because you can create objects outside of the tests and rely on R's copy-onmodify semantics to keep them unchanged between test runs. To clean up other actions you can use regular R functions.

You can run a set of tests just by source () ing a file, but as you write more and more tests, you'll probably want a little more infrastructure. The first 


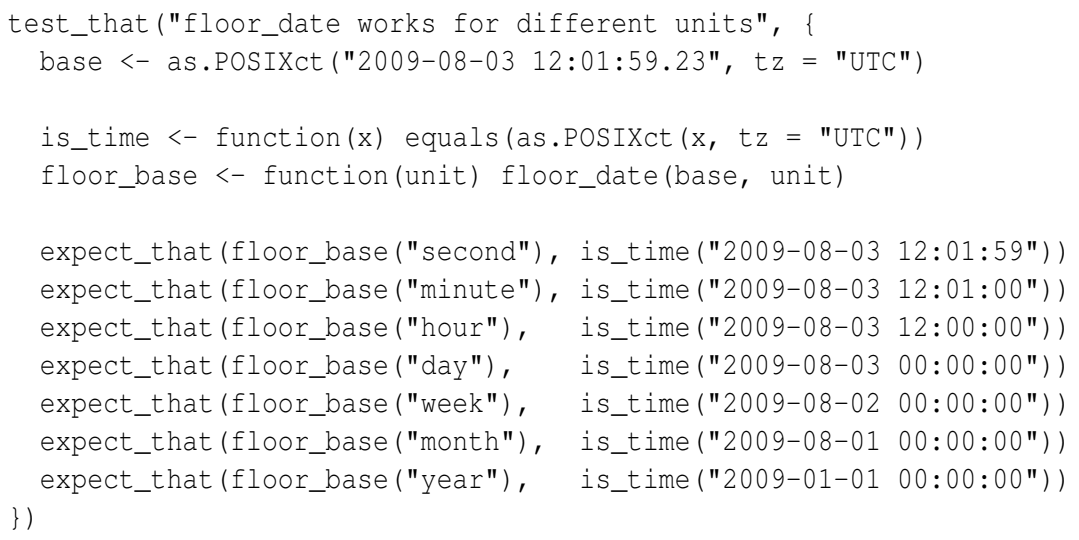

Figure 1: A test case from the lubridate package.

part of that infrastructure is contexts, described below, which give a convenient way to label each file, helping to locate failures when you have many tests.

\section{Contexts}

Contexts group tests together into blocks that test related functionality and are established with the code: context ("My context") . Normally there is one context per file, but you can have more if you want, or you can use the same context in multiple files.

Figure 2 shows the context that tests the operation of the str_length function in stringr. The tests are very simple, but cover two situations where nchar () in base R gives surprising results.

\section{Workflow}

So far we've talked about running tests by source () ing in $\mathrm{R}$ files. This is useful to double-check that everything works, but it gives you little information about what went wrong. This section shows how to take your testing to the next level by setting up a more formal workflow. There are three basic techniques to use:

- Run all tests in a file or directory test_file() or test_dir().

- Automatically run tests whenever something changes with autotest.

- Have R CMD check run your tests.

\section{Testing files and directories}

You can run all tests in a file with test_file (path). Figure 3 shows the difference between test_file and source for the tests in Figure 2, as well as those same tests for nchar. You can see the advantage of test_file over source: instead of seeing the first failure, you see the performance of all tests.
Each expectation is displayed as either a green dot (indicating success) or a red number (indicating failure). That number indexes into a list of further details, printed after all tests have been run. What you can't see is that this display is dynamic: a new dot gets printed each time a test passes and it's rather satisfying to watch.

test_dir will run all of the test files in a directory, assuming that test files start with test (so it's possible to intermix regular code and tests in the same directory). This is handy if you're developing a small set of scripts rather than a complete package. The following shows the output from the stringr tests. You can see there are 12 contexts with between 2 and 25 expectations each. As you'd hope in a released package, all the tests pass.

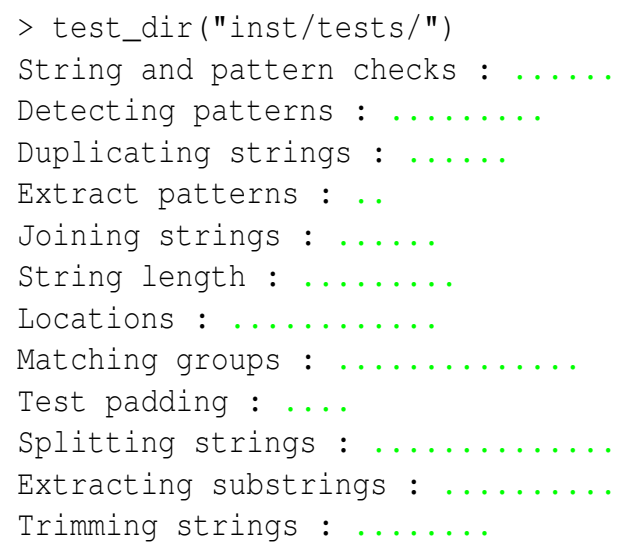

If you want a more minimal report, suitable for display on a dashboard, you can use a different reporter. testthat comes with three reporters: stop, minimal and summary. The stop reporter is the default and stop () s whenever a failure is encountered; the summary report is the default for test_file and test_dir. The minimal reporter prints '. ' for success, ' $E$ ' for an error and ' $F$ ' for a failure. The following output shows (some of) the output from running the stringr test suite with the minimal reporter.

$>$ test_dir("inst/tests/", "minimal") 


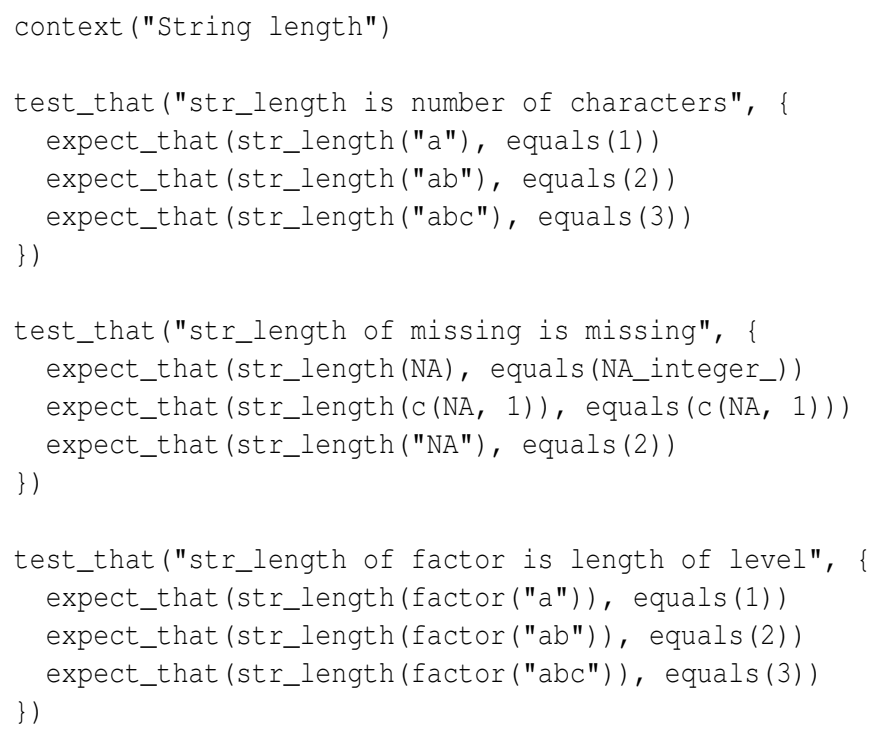

Figure 2: A complete context from the stringr package that tests the str_length function for computing string length.

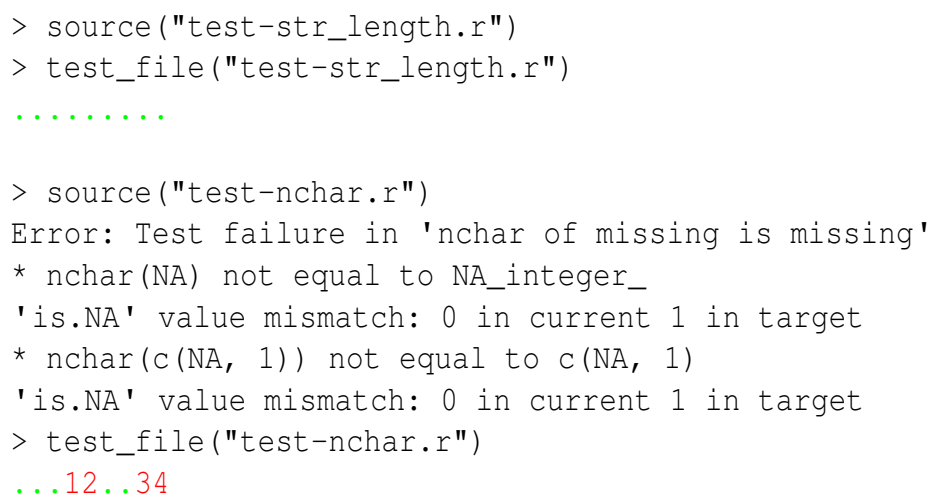

Figure 3: Results from running the str_length context, as well as results from running a modified version that uses nchar. nchar gives the length of NA as 2, and converts factors to integers before calculating length. These tests ensures that str_length doesn't make the same mistakes. 


\section{Autotest}

Tests are most useful when run frequently, and autotest takes that idea to the limit by re-running your tests whenever your code or tests change. autotest() has two arguments, code_path and test_path, which point to a directory of source code and tests respectively.

Once run, autotest () will continuously scan both directories for changes. If a test file is modified, it will test that file; if a code file is modified, it will reload that file and rerun all tests. To quit, you'll need to press Ctrl + Break on windows, Escape in the Mac GUI, or Ctrl + C if running from the command line.

This promotes a workflow where the only way you test your code is through tests. Instead of modify-save-source-check you just modify and save, then watch the automated test output for problems.

\section{R CMD check}

If you are developing a package, you can have your tests automatically run by ' $R$ CMD check'. I recommend storing your tests in inst/tests/ (so users also have access to them), then including one file in tests/ that runs all of the package tests. The test_package (package_name) function makes this easy. It:

- Expects your tests to be in the inst/tests / directory.

- Evaluates your tests in the package namespace (so you can test non exported functions).

- Throws an error at the end if there are any test failures. This means you'll see the full report of test failures and 'R CMD check' won't pass unless all tests pass.

This setup has the additional advantage that users can make sure your package works correctly in their run-time environment.

\section{Future work}

There are two additional features I'd like to incorporate in future versions:
- Code coverage. It's very useful to be able to tell exactly what parts of your code have been tested. I'm not yet sure how to achieve this in $\mathrm{R}$, but it might be possible with a combination of RProf and codetools (Tierney, 2009).

- Graphical display for auto_test. I find that the more visually appealing I make testing, the more fun it becomes. Coloured dots are pretty primitive, so I'd also like to provide a GUI widget that displays test output.

\section{Bibliography}

M. Burger, K. Juenemann, and T. Koenig. RUnit: $R$ Unit test framework, 2009. URL http://CRAN. $\mathrm{R}$-project.org/package=RUnit. $\mathrm{R}$ package version 0.4.22.

P. Grosjean. SciViews-R: A GUI API for R. URL http: //www.sciviews.org/SciViews-R. UMH, Mons, Belgium, 2009.

L. Tierney. codetools: Code Analysis Tools for $R$, 2009. URL http: //CRAN. R-project . org/package= codetools. R package version 0.2-2.

H. Wickham. stringr: Make it easier to work with strings., 2010. URL http://CRAN.R-project.org/ package=stringr. R package version 0.4 .

H. Wickham. testthat: Testthat code. Tools to make testing fun :), 2011. URL http: / /CRAN.R-project.org/ package=test hat. $\mathrm{R}$ package version 0.5 .

H. Wickham and G. Grolemund. lubridate: Make dealing with dates a little easier, 2010. URL http: //www.jstatsoft.org/v40/i03/. R package version 0.1 .

\author{
Hadley Wickham \\ Department of Statistics \\ Rice University \\ 6100 Main St MS\#138 \\ Houston TX 77005-1827 \\ USA \\ hadleyerice.edu
}

